\title{
Congressos e Conferências
}

1985

\section{MARÇO}

25-28

St-Andrews (Escócia)

25-29

Cambridge (GB)

25-29

Coimbra (P)

26-29

Belfast (Irlanda)

31-3/4

Cambridge (GB)

31-3/4

Maynooth (Irlanda)

\section{ABRIL}

1-3

Cambridge (GB)

1-3

New York (USA)

1-4

Strasbourg (F)

9-13

Vienna (Áustria)

14-17

Noordwijkerhout

(Holanda)

14-18

Limbourg (Holanda)

14-19

Limassol (Chipre)

15-18

Bradford (GB)

16-19

Budapeste (Hungria)

15-19

Pretoria (Af. Sul)

\section{5-19}

Melbourne (Austrália)

16-18

Rome (I)

17-21

Paris (F)

16-19

Paris (F)

17-18

Londres (GB)

22-25

Ostende (B)
Annual Chemical Congress of the Royal Society of Chemistry (programme sur demande).

Intern. Meeting of the ESR Group "ESR of Inorgamic Radicals and Metal Ions in Inorganic and Biological Systems",

Ordem dos Engenheiros - Congresso 85

Intern. Symposium "Hydrogen in Metals" (IUPAC).

European Symposium on the Use of Computers in Chemical Engineering.

Second Euchem. Conference on the Electronic Structures of Transition Metal Complexes.

Polymer Liquid Crystals.

1985 Interphex USA 85: The Pharmaceutical et Cosmetic Industries Exposition and Conference

Progrès dans les études par rayons $\mathrm{X}$, grâce au rayonnement synchrotron (symposium).

VIII International Conference on Solid Compounds of Transition Elements.

Intern. Symp. "Biocatalysis in Organic Synthesis".

Colloque "Science et Technologie des Polymères".

2nd Cyprus Conference on New Methods in Drug Research (dépliant s/ demande). Digital Simulation of Time Dependent Chemical Engineering Processes.

6th Symposium on Electroplating

2nd Int. Symp. "Analytical Chemistry in the exploration mining and processing of materials"'.

8th Australian Symp. in analytical Chem.

"Choosing the right method - the pratical chemist's dilemma".

3e Colloque européen sur l'amélioration de la récupération du pétrole.

Colloque intern. sur l'avenir des plastiques et des caoutchoucs dans les transports.

10e Salon intern. "Traitments de surfaces et finition industrielle".

Colloque "Difraction des neutrons aux petits angles par des systèmes organisés". World Filtration Congress IV
22-26

Alsace (F)

24-26

Florence (I)

28-3/5

Miami Beach (USA)

\section{8-4/5}

Burgenstock (Suiça)

\section{MAIO}

6-10

Budapest (Hungria)

8-10

Stockholm (S)

8-10

Baden-Baden (RFA)

8-10

Campinas-SP (Brasil)

9-13

Grasmere (GB)

13-15

Vienne (Austria)

19-25

Strasbourg (F)

20-23

Reims (F)

20-31

Alvor (P)

21-23

Porto (P)

21-25

Beaune (F)

22-23

Paris (F)

27-30

Naples (I)

28-31

Florence (I)

28-31

Yravals (Pyr., F)

\section{JUNHO}

?

New York (USA)

2-4

Londres (GB)

3-5

Uppsala (S)
GECOM XIII-Réunion du Groupe d'Etude de Chimie Organométallique.

Colloque: "Peptides et Transport d'Ions".

Meeting annuel de l'ACS. Thèmes: "Caractérisation des polymères par des techniques de fluorescence. Application analytiques des polymères. Réactions dans les polymères contrôlés par la diffusion. Symp. "Histoire des Polyoléfines".

21e Conférence EUCHEM de Stéróchimie.

XIVe Conference on Silicate Industry and Silicate Science.

Symp. on Properties of Polymers and Drug Delivery.

Anakon'85 "Neue Entwicklungen in der Analytischen Chemie".

III Encontro Nacional de Química Analitica

The 7th Lakeland Heterocyclic Symposium.

12. Kolloquium über Werkstoffanalytik.

XXIIe Semaine d'Etude de la Chimie Organique SECO XXI (inscriptions clôturées le $21 / 12 / 84$ ).

ECPA 85 - Emballage et conservation des produits alimentaires.

Advanced Study Institute on Carbon and Cool Gasification

Materiais 85

GESA XV - Réunion du Groupe d'Etude Structure-Activité-Thèmes: "Le vieillissement: pathologies dégénératives, rôle de l'oxygène, approches thérapeutiques" - "Le génie génétique et ses perspectives thérapeutiques"'. (Nbre inscript. limité).

Colloque sur les Banques de Données en Chimie Inorganique (BDCI).

IUPAC Int. Single Topic Symp. on "Non crystalline order in Plymers".

Journées d'Electrochimie.

Ve Colloque sur la chimie du phosphore.

1st International Symposium on Bioorganic Chemistry (IUPAC).

2e Conf. Intern. sur le Renfocement des Plastiques.

5th Conference on Static Electricity 
3-5

Padoue (I)

3-6

Bloomfield Hills

(Mich., USA)

4-8

Paris (F)

5-7

Londres (GB)

9-17

Frankfurt-au-Man (RFA)

10-12

Würzburg (RFA)

10-14

Schliersee (RFA)

16-22

Monte-Carlo (Monaco)

19-21

Rouen (F)

23-26

Toronto (Canada)

24-25

Stuttgart (RFA)

24-28

Potsdam (NY, USA)

30-4/7

Stockolm (S)

\section{JULHO}

1-4 Schene

1-5

Eindhoven (Holanda)

1-5

Bangor (GB)

1-5

Edimburg (Escócia)

8-11

Prague (Checosl.)

8-12

Cambridge (GB)

15-18

Prague (Checosl.)

11-16

Waterloo (Ont., Can.)

11-15

Kyoto (Japão)

15-19

Reading (GB)

15-19

St-Andrews (Escócia)

18-23

Haya (Holanda)

22-25

Hamilton (Ont., Can.)

22-25

Pretoria (Af. Sul)

23-25

Oxford (GB)

\section{AGOSTo}

11-16

Ontario (Can.)

12-15

Jena (RDA)

18-23

Haia (Holanda)
Journées d'Etude des dels fondus 1985.

Intern. Conference on the Theory of the Structures of non-crystalline Solids (IUPAC).

Bio-Expo 85 - 1er Salon professionnel consacré aux biotechnologies appliquées à la recherche, l'industrie et l'agriculture.

Symp. Intern. sur le chlore "Impact des récents progrès",

Achema 85

5th European Conference on Mixing

7th Intern. Conf. on Computers in Chemical Research and Education - ICCRE.

Mondial Couleur'85 - 5e Congrès de l'Ass. Intern. de la Couleur.

Colloque européen "L'analyse spectrométrique industrielle en ligne".

28th Annual conf. "Food links for progress".

IKT 85 - Conférence internationale du caoutchouc.

5th Intern. Conf. on Surface and Colloïd Science (IUPAC).

Euchem Conference: Computer Modelling in Macromolecular Chemistry.

2e Conférence Internationale sur les films de Langmuir-Blodgett.

7th intern. Symp. on Plasma Chemistry (IUPAC).

Intern. Symp. on Strain and Steric Effects in Organic Chemistry (RSC, Perkin Div.).

9th Intern. Symp. on Column liquid Chromatography.

28th Microsymposium on Macromolecules "Polymer Composites"' (IUPAC).

7 th Intern. Meeting on NMR Spectroscopy.

17e Conférence sur la physique macromoléculaire: "Morphologie des polymères". 10th Internacional Congress of Heterocyclic Chemistry (IUPAC).

3rd IUPAC Symposium on Organometallic Chemistry directed towards organic synthesis.

7th Intern. Symp. on Solute-Solute-Solvent interactions.

5th International Symposium on Novel Aromatic compounds (IUPAC).

30e Symposium International IUPAC sur les Macromolécules.

Faraday Disc.: "Physical Interaction and

Energy Exchange at the Gaz-Solid Interface".

6th Intern. Symp. on Mycotoxins and Phycotoxins.

9th Intern. Symp. "Synthesis in Organic Chemistry” (RSC - Perkin Div.).

Reuth International Congress of Heterocyclic Chemistry.

7th Intern. Symp. on Cationic Polymerization and related processes (IUPAC).

$30 \mathrm{e}$ Symp. Intern. IUPAC sur les Macromolécules.
19-23

Bratislava (Checosl.)

20-22

Bergen (Noruega)

20-24

Tokyo (Japão)

20-24

Salt Lake City, Utah

( U S A )

23-28

Tokyo (Japão)

26-29

Stockolm (S)

\section{SETEMBRO}

2-6

Paris (F)

2-5

Dubrovnik (Jugosl.)

2-5

North Ryde (Australia)

2-5

Paris (F)

2-6

Aix-en-Provence (F)

3-6

Noordwijkerhout

8-13

Viena (Austria)

8-13

Swansea (GB)

9-11

Orléans (F)

9-13

Manchester (GB)

10-13

Leuven (B)

11-13

Ferrara (I)

15-21

Garmisch-Partenk

(RFA)

16-18

Londres (GB)

16-20

Leuven (B)

17-18

Bradford (GB)

17-19

Siofok (Hungria)

17-18

Saint-Denis (F)

17-20

Grenoble (F)

22-28

Woods Hole (Mass., U S A )

25-27

Dublin (Irlanda)

25-27

Nancy (F)

25-27

Bordeaux (F)

30-3/10

Venesa (I) 8th Intern. Conference on Thermal Analysis (IUPAC).

Intern. Symp. on Reliable Flow of Particulate Solids.

Symp. Intern.: "Les fibres: Science et Technologie".

International Conference on Defects in Insulating Crystals.

8th Intern. Conf. on Chemical Education: "Widening the scope of chemitry" (IUPAC).

Intern. Symp. on New Trends in the Photochemistry of Polymers.

Vth International Symposium on Marine Natural Products.

Intern. Symp. on Applications of Mathematical Concepts to Chemistry (IUPAC).

Colloque "Propriétés électriques, optiques et acoustiques des polymères".

Colloque intern.: "Comportement mécanique et physique des matériaux sous sollicitations dynamiques"'.

ESOC IV - 4e Symposium Européen de Chimie Organique.

3rd Noordwijkerhout Symp. "Innovative Approaches in Drug Research" (dépliant sur demande).

XIIth Intern. Conference on Organometallic Chemistry.

10th International Symposium on Mass Spectrometry.

Conférence Internationale "Science of Ceramics 13"'.

30th Internactional Congress of Pure and Applied Chemistry.

5th International Conference: Chemistry for Protection of the Enviroment.

Workshop on Electrochemical Corrosion Testing with Special Consideration of Practical Applications.

Colloquium Spectrospicum International. XXIV. 11th Intern. Conf. on Atomic Spectroscopy.

2nd European Conference on Coal Liquid Mixtures.

International Conference on the Applications of the Mössbauer Effect.

Advances in Process Control.

Conference on "Temporary Corrosion Preventives and Anticorrosive Lubricants". ler Colloque francophone sur I'Ignifugation des polymères.

3e Congrès Européen sur les glucides.

CHEMRAWN IV (Chemical Resources of the Global Oceans (IUPAC).

Conference on Fine Chemical Processing.

Bio +85 . Rencontres Internationales de bio-industries.

Colloque "Développement de la Science et de la Technologie des matériaux composites".

$2 \mathrm{e}$ Congrès mondial "Métallurgie et applications du cobalt". 
30-2/10

Bâle (Suíça)

\section{OUTUBRO}

9-11

Antwerp (B)

15-18

Kyoyo (Japão)

15-19

Pretoria (Af. Sul)

28-31

Tützing (RFA)

28-1/11

Sydney (Australia)

23-25

Lisboa (P)

\section{NOVEMBRO}

3-7

10-13

Shangai (China)

19-21

Nice (F)

26-29

Zurich (Suiça)

\section{DEZEMBRO}

? São Carlos-SP (Brasil)

1986

\section{JANEIRO}

? Cadiz (Espanha)

\section{ABRIL}

16-18 Nuremberg (RFA)

22-24

Noordwijkerhout (Holanda)

\section{MAIO}

11-16

Helsingor (DK)

19-21

Philadelp

19-22

Brigton (GB) and Conference.

International Symposium on Organic Chemistry of Medical Natural Products. 7th European Congress of Corrosion (EFC).

Interpleex Europe 85

III Seminário sobre Quimica Marinha.

Réunion commune des Sociétés Biochimiques de France, RFA et Suisse.

6th International Conference Large Chemical Plants.

Symp. Intern. "Chimie analytique (-exploitation minière et traitemet de la matière".

Tützing Symp. "Liquid Crystals in Mate-

2. ${ }^{\circ}$ Simpósio Nacional de Produção de Novas proteinas e Utilização de Recursos Inexplorados.

II Simpósio Brasileiro de Química Teórica.

1st World Congress on Particle Technology.

4th Intern. Conf. on Fluid Properties and Phase Equilibria - Process Design.

ISCRE 9 - Intern. Symp. on chemical Reaction Engineering.

5th BEAMA International Insulation Conference.

\section{ANATECH 86}

\section{JUNHO}

3-6

Munich (RFA)

Analytica 86 .

JULHO

14-18

Lisboa (P)

20-26

Bristol (GB)

21-25

Univ. Califórnia, Berkeley (USA)

27-2/8

Lisboa (P)

\section{AGosto}

?

Campinas (SP, Brasil)

10-15

Moscou (URSS)

10-17

Ottawa (Can.)

17-22

Tokyo (Japão)

17-22

(Holanda)

17-23

La Haye (P-B)

21-2/9

Munich (RFA)

25-29

Antuérpia (B)

24-29

Tokyo (Japão)

\section{SETEMBRO}

1-5

Beijung (China)

1-7

Bonn (RFA)

8-12

Padoue (I)

15-19

Cannes (F)

15-19

Berlin (RFA)

21-25

Tokyo (Japão) dynamics. Chemistry. gins of Life. (ISEC). Chemistry. mistry. neering.
IUPAC Conference on Chemical Thermo-

International Conference on Analytical

8th International Conference on the Ori-

5th Meeting of the International Society for the Study of the Origin of Life.

XI IUPAC Conference on Photochemistry

Recent Developments in Organic NMR Workshop Brasil-USA.

Sixth International Conference on Organic Synthesis (IUPAC).

6th International Pesticide Chemistry Congress (IUPAC).

7th Intern. Zeolite Conference.

15th IUPAC International Symposium on the Chemistry of Natural Products.

15 the Intern. Symp. on the Chemistry of Natural Products.

Intern. Solvent Extraction Conference

10th International Symposium on Microchemical Techniques.

8th IUPAC Conference on Physical Organic Chemistry.

International Conference on Nuclear and Radiochemistry

International Congress on Phosphorus

5th Intern. Conf. on Organometallic and Coordination Chemistry of Germanium. Tin and Lead.

5th Intern. Symp. on Loss Prevention and Safety Promotion in the Process Industry. IXth Intern. Symposium on Medicinal Che-

World Congress III of Chemical Engi- 